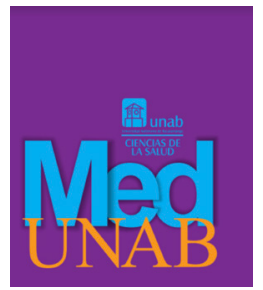

REVISTA DE LA FACULTAD

DE CIENCIAS DE LA SALUD

\title{
Incidencia de cáncer de colon y recto en Bucaramanga, Colombia 2008 - 2012
}

Incidence of colorectal cancer in Bucaramanga, Colombia 2008 - 2012

Incidência de câncer colorretal em Bucaramanga, Colômbia, no período 2008 - 2012

Claudia Janeth Uribe-Pérez, MD., Esp., MSc. ${ }^{1}$ iD, July Juliana Blanco-Quintero, MD. ${ }^{2}$ (D) Lina Marcela Bello-Zapata, MD. ${ }^{2}$ (D)

1. Médica, Especialista en Patología, Maestría en Educación Médica, Directora del Registro Poblacional de Cáncer del Área Metropolitana de Bucaramanga, Directora del Grupo de Investigación Estudio Genético de Enfermedades Complejas, Universidad Autónoma de Bucaramanga. Floridablanca, Santander, Colombia.

2. Médica, Integrante del Grupo de investigación Estudio Genético de Enfermedades Complejas, Universidad Autónoma de Bucaramanga. Floridablanca, Santander, Colombia.

Correspondencia: July Juliana Blanco, Médico, Universidad Autónoma de Bucaramanga; código postal 68003. E-mail: jblanco144@unab.edu.co

Cómo citar: Uribe-Pérez CJ, Blanco-Quintero JJ, Bello-Zapata LM. Incidencia de cáncer de colon y recto en Bucaramanga, Colombia 2008 - 2012. MedUNAB. 2019;22(1):16-23. doi: 10.29375/01237047.2711

\section{INFORMACIÓN ARTÍCULO}

Artículo recibido: 07 de julio de 2017

Artículo aceptado: 11 de diciembre de 2018

DOI: https://doi.org/10.29375/01237047.2711

\section{RESUMEN}

Introducción. El cáncer es la segunda causa de muerte en el mundo, el cáncer colorrectal la cuarta neoplasia más frecuente. Debido al aumento progresivo, se hace importante conocer el impacto de esta neoplasia en el área metropolitana de Bucaramanga. El objetivo es describir la incidencia y características sociodemográfica del cáncer colorrectal en el Área Metropolitana de Bucaramanga durante el 2008 - 2012.

Metodología. Se realizó un estudio poblacional descriptivo transversal basado en casos incidentes de cáncer colorrectal del Área Metropolitana de Bucaramanga. La información de nuevos casos ocurridos durante 2008 - 2012 se obtuvo del Registro Poblacional de cáncer. Los criterios de inclusión fueron: pacientes con lesión primaria e infiltrante de colon y recto, sin límite de edad o sexo. Se excluyeron los casos que 
correspondían a recaída, recidiva o metástasis, cuya morfología comprendiera el tipo linfoma. Posteriormente se calcularon las tasas crudas y estandarizadas por edad y sexo mediante el programa CanReg5.

Resultados. Se obtuvieron 805 casos nuevos de cáncer colorrectal, con una incidencia de 14.3 casos en hombres y 13.5 casos en mujeres por 100,000 habitantes; la edad promedio de diagnóstico fue de 64 años para ambos sexos. Conclusiones. La incidencia de cáncer colorrectal ha ido en aumento con respecto a quinquenios anteriores en el Área metropolitana de Bucaramanga, afectando en mayor proporción a la población adulta mayor masculina y convirtiéndose en un reto de salud pública que demanda mayores medidas de prevención y estudios de esta patología.

Palabras clave:

Incidencia; Neoplasias; Colon; Recto; Neoplasias Colorrectales; Colombia.

\section{ABSTRACT}

Introduction. Cancer is the second cause of death in the world, with colorectal cancer being the fourth most frequent neoplasia. Due to a progressive increase, it is important to know the impact of this neoplasia in the Metropolitan Area of Bucaramanga. The objective is to describe the incidence and sociodemographic characteristics of colorectal cancer in the Metropolitan Area of Bucaramanga during 2008 - 2012.

Methodology. A cross-sectional descriptive population study based on incident cases of colorectal cancer from the Metropolitan Area of Bucaramanga was conducted. The information of new cases that occurred during 2008 - 2012 was obtained from the Population Registry of Cancer. The inclusion criteria were: patients with primary and infiltrating lesions of the colon and rectum, with no age or sex limit. Cases that corresponded to relapse, recurrence or metastasis, and whose morphology included the type of lymphoma were excluded. Subsequently, the crude and standardized rates for age and sex were calculated using the CanReg5 program.

Results. 805 new cases of colorectal cancer were obtained, with an incidence of 14.3 cases in men and 13.5 cases in women per 100,000 inhabitants; the average age of diagnosis was 64 years for both sexes.

Conclusions. The incidence of colorectal cancer has increased compared to previous five-year periods in the Metropolitan Area of Bucaramanga, affecting older adult male population to a greater extent, becoming a public health challenge, which requires more prevention measures and studies of this pathology.

Keywords

Incidence; Neoplasia; Colon; Rectal; Colorectal Neoplasia; Colombia.

\section{RESUMO}

Introdução. O câncer é a segunda causa de morte no mundo, sendo o câncer colorretal a quarta neoplasia mais frequente. Devido ao aumento progressivo, é importante conhecer o impacto dessa neoplasia na Região Metropolitana de Bucaramanga. O objetivo deste trabalho é descrever a incidência e as características sociodemográficas do câncer colorretal na Região Metropolitana de Bucaramanga no período de 2008 a 2012.

Métodos. Foi realizado um estudo descritivo e transversal de base populacional baseado em casos incidentes de câncer colorretal na Região Metropolitana de Bucaramanga. A informação sobre os novos casos ocorridos no período de 2008 a 2012 foi obtida no Registro Populacional de câncer. Os critérios de inclusão foram: pacientes com lesão primária e infiltrativa de cólon e reto, sem limite de idade nem distinção de sexo. Foram excluídos os casos de recorrência, recidiva ou metástase, cuja morfologia incluía o tipo de linfoma. Posteriormente as taxas brutas e padronizadas por idade e sexo foram calculadas usando o Software CanReg5.

Resultados. Houve 805 novos casos de câncer colorretal, com uma incidência de 14.3 
casos em homens e 13.5 casos em mulheres por 100,000 habitantes; A idade média do diagnóstico foi de 64 anos para ambos os sexos.

Conclusão. A incidência de câncer colorretal vem aumentando em relação aos quinquênios anteriores na Região Metropolitana de Bucaramanga, afetando em maior proporção os homens idosos e tornando-se um desafio de saúde pública que demanda maiores medidas de prevenção e mais estudos dessa patologia.

Palavras-chave:

Incidência; Neoplasias; Colo; Reto; Neoplasias Colorretais; Colômbia.

\section{Introducción}

El cáncer colorrectal (CCR) es una neoplasia que se caracteriza por la proliferación celular descontrolada que progresa a malignidad desde el colon ascendente hasta la región rectal. Este se agrupa en diferentes tipos de cáncer según su origen morfológico; por una parte, los adenocarcinomas representan más del $95 \%$, mientras que los tumores menos frecuentes son de tipo: carcinoides, estromales, sarcomas, linfomas y del tejido conectivo (1).

Entre los factores de riesgo para el desarrollo de esta enfermedad están los modificables y no modificables; en los primeros encontramos los estilos de vida: dietas con consumo de 100 gr/día de carnes rojas y 50 gr/día de carne procesada aumentan un $17 \%$ y $18 \%$ respectivamente el riesgo de padecer CCR $(2,3)$, también el consumo excesivo de alcohol, tabaquismo, poca actividad física y obesidad. En cuanto a los factores no modificables se encuentra la edad, antecedentes personales de pólipos adenomatosos con o sin displasia, síndromes genéticos de poliposis familiares (4), antecedente de enfermedad inflamatoria del intestino (enfermedad de Crohn o colitis ulcerosa) y antecedentes familiares de primer grado; este factor presenta mayor riesgo para las personas, ya que 1 de cada 5 personas que padece CCR tiene otros familiares que han sido afectados por esta enfermedad (1).

El cáncer es una de las principales causas de morbilidad y mortalidad en el mundo; por ejemplo, en el 2015 fue la segunda causa de muerte según la Organización mundial de la salud (OMS) (5), ocasionando 8.8 millones de defunciones. Respecto al año 2012, el CCR ocupó el cuarto lugar entre las lesiones neoplásicas más frecuentes en el mundo para ambos sexos y Australia fue el país que tuvo mayor incidencia con tasas de 38.4 / 100,000 habitantes. Cabe mencionar que a nivel mundial es el quinto cáncer más mortal para ambos sexos; en hombres es superado por el cáncer de pulmón y próstata, y en mujeres por el cáncer de mama (6).
Para el 2012, según Globocan (6), el CCR ocupó en Colombia el quinto lugar de los tumores malignos más frecuentes para ambos sexos con una tasa de incidencia de 12.9 casos / 100,000 habitantes y tuvo el mismo lugar de frecuencia en mortalidad con tasas de hasta 7.2 casos / 100,000 habitantes. En un reporte del Instituto Nacional de Cancerología (7) para los años 2007 - 2011, el CCR mostró una tasa de incidencia de 12.2 casos / 100,000 habitantes para ambos sexos y una tasa de mortalidad de 6 casos / 100,000 habitantes. En Cali, según datos obtenidos del Registro Poblacional de Cáncer para el 2008 - 2012, se encontraron cifras mayores respecto a nivel nacional, con tasas de incidencia para hombres de 16.2 y para mujeres de 13.7 casos / 100,000 habitantes (8).

En el Área Metropolitana de Bucaramanga (AMB), durante el 2003-2007 y de acuerdo a datos obtenidos del Registro poblacional de cáncer del Área metropolitana de Bucaramanga (RPC-AMB), el CCR ocupó el tercer puesto en orden de frecuencia para ambos sexos; en hombres fue precedido por cáncer de próstata y estómago, y en mujeres por cáncer de mama y cuello uterino, con tasas de incidencia de 13.3 y de 12.2 casos / 100,000 respectivamente (9).

Estadios tempranos del CCR usualmente no presentan síntomas o se presentan de manera inespecífica como: rectorragia, hematoquecia, cambio del hábito intestinal o forma de las heces, dolor en hemiabdomen inferior, inapetencia y pérdida de peso, por lo que se hace importante el tamizaje de esta patología para detectar el cáncer en estadios iniciales (10). En Colombia no hay estudios específicos respecto a la cobertura de tamización del CCR dentro del Sistema General de Seguridad Social en Salud. La prueba de rutina para tamización que se realiza en la actualidad es el test de Guayaco que se encuentra incluida en el Plan Obligatorio de Salud; tampoco hay estudios particulares recientes sobre carga de enfermedad en términos de años de vida saludable perdidos con relación al cáncer de colon y recto en Colombia (11). 
Debido a la tendencia ascendente en la incidencia del CCR en el mundo y en el país, se considera necesario continuar y ampliar el estudio descriptivo de esta neoplasia en el AMB, cuyos datos promuevan y ayuden a garantizar que se generen políticas para la disminución de esta enfermedad.

\section{Metodología}

Se realizó un estudio poblacional descriptivo transversal, basado en información de casos nuevos de cáncer de colon y recto en el AMB (incluye a Bucaramanga, capital del departamento de Santander y tres municipios contiguos; a saber, Floridablanca, Piedecuesta y Girón) con una población total de 1,074,918 habitantes, según proyecciones del Departamento Administrativo Nacional de Estadística (DANE) para el 2010 (12). La información de los nuevos casos de CCR desde enero de 2008 hasta diciembre de 2012 fueron recolectados por búsqueda activa en el RPC-AMB. Se extrajeron datos de cada paciente con base en un cuestionario diseñado y diligenciado por el RPC-AMB; para ello se utilizaron fuentes de información gubernamentales y de laboratorios de patología, como edad, género, fecha de nacimiento, fecha de defunción, régimen de salud, lugar de residencia del paciente, fecha del diagnóstico, método diagnóstico, localización de cáncer, histología de este y grado de diferenciación.

Posteriormente, esa información se recolectó y se sistematizó durante el desarrollo del presente estudio mediante el programa CanReg5 e IARCTools, diseñado por la International Agency for Research on Cancer (IARC) (13) para los RPC; estas herramientas permitieron la disminución de sesgos de selección e información. Además, se cumplió con los lineamientos de confidencialidad y compromisos éticos de la investigación establecidos por la IARC. Se quiere especificar que la intención de este estudio fue continuar la descripción detallada y sucesiva de incidencia por quinquenios del $\mathrm{AMB}$, cuyas últimas publicaciones fueron los casos reportados hasta el año de 2007 (9).

Mediante diferentes métodos diagnósticos se obtuvieron 813 nuevos casos de cáncer colorrectal. Los criterios de inclusión para el análisis fueron pacientes con lesión maligna primaria infiltrante, localizada en las regiones correspondientes al código de diagnóstico según la clasificación internacional de enfermedades para oncología (CIE-O) del C18 al C20, que comprenden lesiones neoplásicas desde el colon hasta el recto respectivamente, cuya morfología comprendiera adenocarcinomas, carcinomas, leiomiosarcomas, neoplasias malignas, sarcomas, tumores carcinoides y tumor rabdoide de colon y recto dentro del periodo mencionado; no se aplicaron límites de edad o sexo. Los criterios de exclusión del estudio fueron pacientes con morfología de linfomas, ya que esta difiere de la fisiopatología del CCR, además de los casos que corresponden a recaída, recidiva o metástasis.

Para la estimación de tasas de incidencia cruda (TC) y tasas de incidencia estandarizada (TEE) por edad y sexo, se tuvo en cuenta la proyección del DANE (12) para la población del AMB del periodo de estudio y la población estándar mundial de Segi modificada por Doll y colaboradores (14), las cuales se calcularon por método directo y CanReg5 para su interpretación.

\section{Resultados}

De los 813 pacientes registrados con cáncer infiltrante de colon y recto durante el periodo de estudio, se excluyeron 8 casos con morfología correspondiente a linfoma; en total se obtuvieron 805 casos con diagnóstico definitivo de CCR maligno. Un $56 \%$ de los casos se presentó en mujeres y la edad promedio al momento del diagnóstico en ambos sexos fue de 64 años. Una gran parte de los habitantes residía en el municipio de Bucaramanga (57\%), seguido de Floridablanca (24\%), cuyo $50 \%$ pertenecía al estrato socioeconómico medio y un $61 \%$ de esa misma población al régimen contributivo (Tabla 1).

El $90 \%$ de los casos se obtuvo por verificación histológica, seguido por certificados de defunción (6 $\%)$. Las localizaciones anatómicas más frecuentes en ambos sexos según códigos del CIE-O fueron recto $(25$ $\%)$, colon sigmoides (16\%), colon ascendente (12\%) (Figura 1), y el tipo morfológico más representativo fue el adenocarcinoma ( $84 \%)$.

Se dio mayor número de casos de cáncer de colon (69.4 \%) versus cáncer de recto; el primero fue predominante en mujeres $(58.8 \%)$, mientras que para el cáncer de recto se presentó igual número de casos en hombres y mujeres.

La tasa cruda de CCR fue mayor en mujeres (16.2 casos / 100,000 habitantes) que en hombres (13.7 casos / 100,000 habitantes). Se evidenció un incremento de casos de CCR a partir de los 60 - 64 años, con tasas máximas de incidencia entre los 70 - 74 años y mayores de 80 años. (Figura 2). La TEE de CCR en hombres fue de 14.3 casos / 100,000 habitantes y en mujeres 13.5 casos / 100,000 habitantes; además de esto se mostró significativamente mayores TEE a partir de los 60 años en ambos sexos (Tabla 2). 
Tabla 1. Variables sociodemográficas por sexo en casos de cáncer colorrectal en el AMB durante el 2008 - 2012

\begin{tabular}{lll}
\hline $\begin{array}{l}\text { Variables sociodemográficas de cáncer de } \\
\text { colon y recto }\end{array}$ & $\mathrm{n}$ Mujeres (\%) & $\mathrm{n}$ Hombres (\%) \\
\hline Muestra completa $\mathrm{n}=805$ & $452(56)$ & $353(44)$ \\
\hline Municipio & $\mathrm{n}$ Mujeres (\%) & $\mathrm{n}$ Hombres (\%) \\
\hline Bucaramanga & $257(32)$ & $203(25)$ \\
\hline Floridablanca & $101(13)$ & $86(11)$ \\
\hline Girón & $48(6)$ & $34(4)$ \\
\hline Piedecuesta & $41(5)$ & $26(3)$ \\
\hline Desconocido & $5(1)$ & $4(0)$ \\
\hline Estrato socieconómico & $\mathrm{n} \mathrm{Mujeres} \mathrm{( \% )}$ & $\mathrm{n} \mathrm{Hombres} \mathrm{( \% )}$ \\
\hline Bajo (1,2) & $95(12)$ & $69(9)$ \\
\hline Medio (3,4) & $227(28)$ & $179(22)$ \\
\hline Alto (5,6) & $40(5)$ & $36(4)$ \\
\hline Desconocido & $90(11)$ & $69(9)$ \\
\hline Régimen de salud & $\mathrm{n} \mathrm{Mujeres} \mathrm{( \% )}$ & $\mathrm{n} \mathrm{Hombres} \mathrm{( \% )}$ \\
\hline Contributivo & $269(33)$ & $222(28)$ \\
\hline Subsidiado & $112(14)$ & $76(9)$ \\
\hline Excepción & $47(6)$ & $34(4)$ \\
\hline Prepagada & $2(0,2)$ & $3(0.4)$ \\
\hline Desconocido & $14.7)$ \\
\hline Particular & $(1)$ & $6.7)$ \\
\hline
\end{tabular}

\section{Discusión}

En nuestro grupo de estudio, se presentaron las TEE de cáncer CCR del 2008 - 2012, tiempo en el que se evidenció un aumento de casos nuevos de CCR respecto al periodo previamente estudiado (2003 - 2007) del AMB (9), pasando de 13.3 casos / 100,000 en hombres y 12.2 casos / 100,000 en mujeres a TEE de 14.3 y 13.5 respectivamente. De igual forma, el CCR se mantuvo como el tercer cáncer más incidente para ambos sexos (15). Asimismo, permaneció durante estos periodos consecutivos con mayor incidencia de CCR en hombres, como también mayores tasas de incidencia a partir de los 65 años. Este comportamiento fue el mismo registrado en Cali en cuanto a edad y predominio de CCR en los hombres; sin embargo, Cali presentó mayor número de casos nuevos de CCR para el mismo periodo respecto al presentado en el AMB, con TEE de 15.9 en hombres y 13.7 casos / 100,000 habitantes en mujeres (8).

A nivel nacional la incidencia estandarizada en ambos sexos de CCR fue de 12.9 casos / 100,000 habitantes según Globocan (6); esta cifra es superada por la población del AMB en el periodo 2008 - 2012 y difiere de las TEE a nivel mundial donde la población del AMB presenta menores tasas de incidencia. 
*Otras localizaciones

C18.4 Colon transverso

C19.9 Union rectosigmoidea

C18.6 Colon descendente

C18.0 Ciego

C18.2 Colon ascendente

C18.7 Colon sigmoide

C18.9 Colon (sin localización especifica)

C20.9 Recto

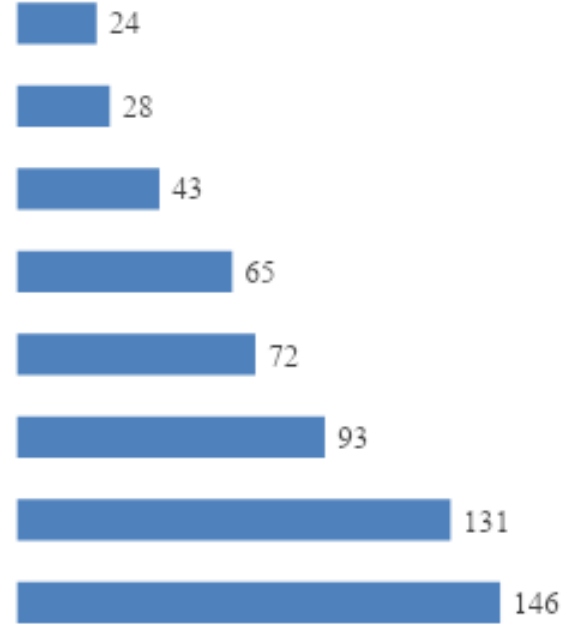

146

Figura 1. Localizaciones más frecuentes según CIE - O de CCR en ambos sexos durante 2008 - 2012 en el AMB. *Otras localizaciones incluyen: C18.5 Ángulo esplénico del colon, C18.8 Lesión sobrepuesta de colon, C18.3 Ángulo hepático del colon

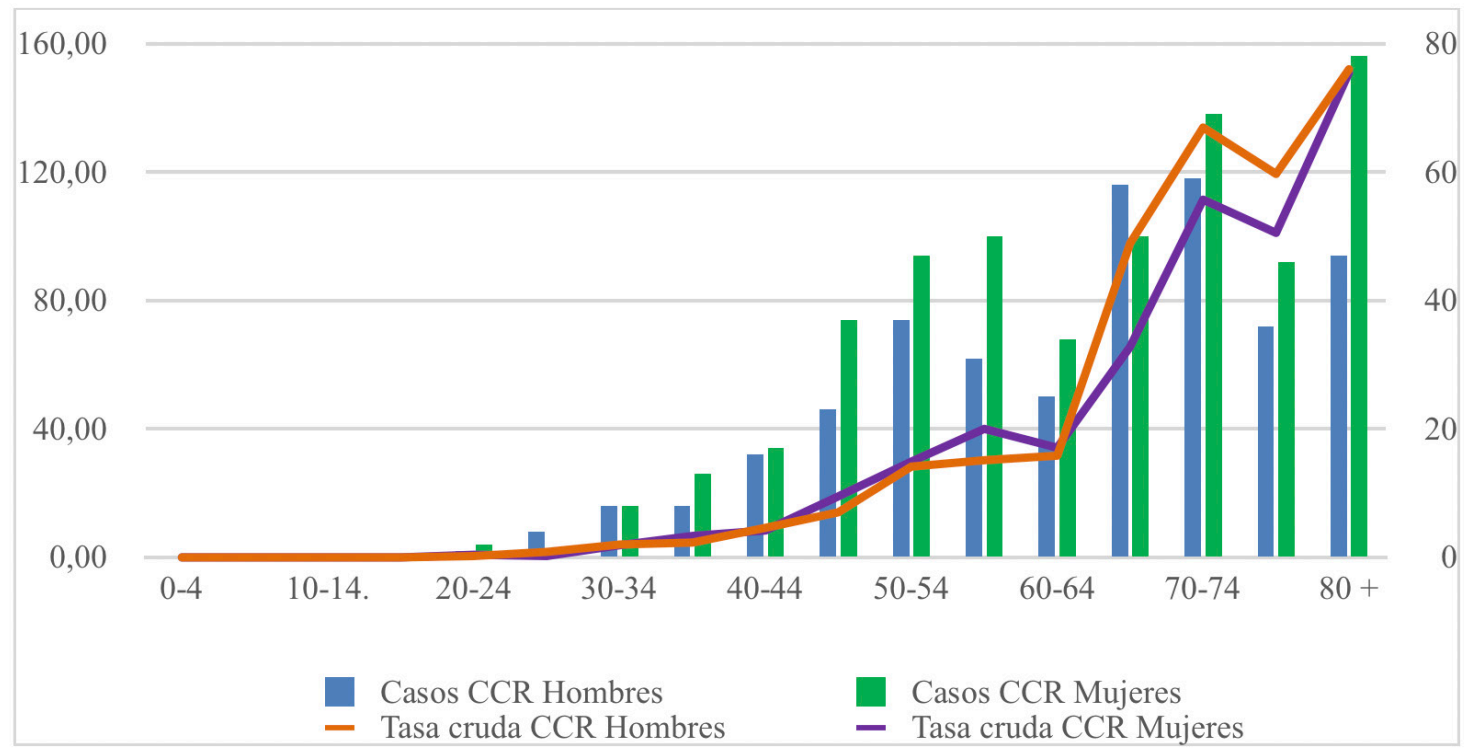

Figura 2. Tasas crudas y número de casos de cáncer colorrectal (CCR) en hombres y mujeres en el AMB durante $2008-2012$. 
Tabla 2. Tasas de incidencias crudas y estandarizadas de cáncer colorrectal en menores y mayores de 60 años en el AMB durante 2008 - 2012.

\begin{tabular}{lllll}
\hline & \multicolumn{4}{c}{ Cáncer colorrectal } \\
\hline Hombres & $\mathbf{n}$ & $\mathbf{\%}$ & TC & TEE \\
\hline$<50$ años & 60 & 7.1 & 2.6 & 2.1 \\
\hline$>50$ años & 293 & 34.5 & 120.4 & 12.2 \\
\hline Total & 353 & 41.5 & 13.7 & 14.3 \\
\hline Mujeres & $\mathbf{n}$ & $\mathbf{\%}$ & TC & TEE \\
\hline$<50$ años & 78 & 9.2 & 3.6 & 2.4 \\
\hline$>50$ años & 374 & 44.0 & 60.6 & 11.2 \\
\hline Total & 452 & 53.2 & 16.2 & 13.5 \\
\hline
\end{tabular}

El comportamiento progresivo del CCR en el AMB se podría asociar con el desarrollo económico de la población, pues estudios recientes han encontrado que, en países de alto ingreso, los habitantes presentan mayor sedentarismo, obesidad, tabaquismo, alcoholismo, dietas altas en alimentos procesados, grasas y carnes rojas; todos estos son factores que están relacionados con mayor riesgo de CCR $(16,17)$. Este estilo de vida en el AMB se ve reflejado en la última encuesta de situación nutricional del país, ya que el consumo de grasas saturadas en las zonas urbanas duplica el consumo observado en las zonas rurales (30\% vs. $16 \%$ respectivamente) (18).

Teniendo en cuenta que el CCR se desarrolla bajo factores de riesgo modificables, tomar medidas de prevención primaria y detección precoz es de vital importancia para reducir su incidencia (3). Debido a esto se han propuesto múltiples esquemas de tamización, como lo propuesto en la Guía 2018 de la Sociedad Americana contra el Cáncer, en la que plantean iniciar tamizaje a partir de los 45 años en pacientes con riesgo promedio de CCR (19). De la misma forma se plantea realizar por sociedades médicas en Colombia, sin poder llegar aún a un consenso reproducible para nuestra población (11). Según la guía de práctica clínica realizada en el 2015 por la Asociación Colombiana de Gastroenterología (20), se considera realizar tamización anual a partir de los 50 años en pacientes con riesgo promedio de CCR mediante el Test de Guayaco, ya que este se encuentra incluido en el Plan Obligatorio de Salud y tiene un costo razonable; esto se plantea con la recomendación de individualizar e identificar aquellos pacientes que tienen factores de alto riesgo y que, independientemente de la edad, deben ser estudiados mediante métodos de tamizaje invasivos, como la colonoscopia que es el Gold estándar para detección de CCR (16). No obstante, la colonoscopia no es la herramienta adecuada como método de tamizaje en la población en general, dado la mala adherencia, alto riesgo de complicaciones y costos. Esto último se ve reflejado, según la clasificación de afiliación al Sistema General de Seguridad Social, cuando los pacientes que pertenecen al régimen contributivo son quienes tienen mayor acceso a servicios médicos y de tamización respecto aquellos que pertenecen al régimen subsidiado o vinculados (16).

\section{Conclusiones}

Este estudio demuestra que el CCR es una enfermedad de interés para la salud pública, ya que presenta una incidencia ascendente en el AMB, con mayor impacto en el adulto mayor. Cabe resaltar que al respecto hay pocos estudios y guías clínicas actualizadas que midan el impacto a nivel social, económico y morbimortalidad, por lo que se deben implementar estrategias reales con el fin de mejorar estilos de vida saludables y medidas de detección temprana unificadas que estén al alcance de toda la población.

\section{Conflictos de Interés}

Se declara que durante la realización de este manuscrito no existieron conflictos de interés.

\section{Agradecimientos}

Los resultados se han obtenido gracias al apoyo de la Universidad Autónoma de Bucaramanga UNAB, y el 
Instituto Nacional de Cancerología quienes financian al RPC-AMB.

\section{Referencias}

1. American Cancer Society. Cáncer Colorrectal [Internet]. 2018 [citado marzo 2018]. Recuperado a partir de: https://www.cancer.org/es/cancer/cancer-de-colon-o-recto/acerca/que-es-cancer-de-colon-o-recto. html.

2. Organización mundial de la salud. Carcinogenicidad del consumo de carne roja y de la carne procesada [Internet]. 2015 [citado marzo 2018]. Recuperado a partir de: http://www.who.int/features/qa/cancer-redmeat/es/.

3. American Cancer Society. Colorectal Cancer Causes, Risk Factors, and Prevention [Internet]. 2018 [citado marzo 2019]. Recuperado a partir de: https://www.cancer.org/content/dam/CRC/ PDF/Public/8605.00.pdf

4. Kanth P, Grimmett J, Champine M, Burt R, Samadder N. Hereditary Colorectal Polyposis and Cancer Syndromes: A Primer on Diagnosis and Management. Am J Gastroenterol. 2017; 112:1509-1525. doi: 10.1038/ ajg.2017.212

5. Organización mundial de la salud. Cáncer [Internet]. 2018 [citado marzo 2018]. Recuperado a partir de: http://www.who.int/mediacentre/factsheets/fs297/es/.

6. Globocan. Cancer Today [Internet]. 2012 [citado diciembre 2017]. Recuperado a partir de: http://gco.iarc. fr/today/fact-sheets-populations.

7. Pardo C, Cendales R. Instituto Nacional de Cancerología - Colombia [Internet]. 2015 [citado marzo 2018]. Recuperado a partir de: http://www.cancer.gov. co/files/libros/archivos/incidencia1.pdf

8. Bravo LE, García LS, Collazos P, Carrascal E, Ramírez $\mathrm{O}$, Cortés A, et al. Información fiable para el control del cáncer en Cali, Colombia. Colomb Med (Cali). 2018; 49(1): 23-34. doi: 10.25100/cm.v49i1.3689

9. Uribe C, Osma S, Herrera V. Cancer incidence and mortality in the Bucaramanga metropolitan area, 2003-2007. Colomb Med (Cali). 2013; 43(4):290 297

10. American Cancer Society. Cancer Facts and Figures 2017 [Internet]. 2017 [citado marzo 2019]. Recuperado a partir de: https://www.cancer.org/content/dam/ cancer-org/research/cancer-facts-and-statistics/annual-cancer-facts-and-figures/2017/cancer-facts-andfigures-2017.pdf

11. Instituto Nacional de Cancerología, Ministerio de Salud y Protección Social. Guía de práctica clínica para pacientes con diagnóstico de cáncer de colon y recto. Guía No.2013 - 20 [Internet]. 2013 [citado marzo 2018]. Recuperado a partir de: http://gpc.minsalud. gov.co/gpc sites/Repositorio/Conv 500/GPC cancer_colon/GC_Comple_Ca_Colon.pdf

12. DANE. Proyecciones de población municipales por área [Internet]. 2005 [citado diciembre 2017]. Recuperado a partir de: https://www.dane.gov.co/files/ investigaciones/poblacion/proyepobla06_20/ProyeccionMunicipios2005 2020.xls

13. International Agency for Research on Cancer. CanReg5 [Internet]. 2018 [citado marzo 2018]. Recuperado a partir de: http://www.iacr.com.fr/ index.php?option $=$ com_content $\&$ view $=$ category\&id $=68 \&$ Itemid $=445$

14. Ahmad O, Boschi-Pinto C, López A. World Health Organization [Internet]. 2001 [citado marzo 2018]. Recuperado a partir de: http://www.who.int/healthinfo/paper31.pdf

15. Uribe CJ, Serrano SE, Hormiga CM. Cancer incidence and mortality in Bucaramanga, Colombia. 2008-2012. Colomb Med (Cali). 2018;49(1):73-80. doi: $10.25100 / \mathrm{cm} . v 49 \mathrm{il} .3632$.

16. Colorectal Cancer Facts \& Figures 2017-2019. American Cancer society [Internet]. 2019 [citado febrero 2019]. Recuperado a partir de: https:// www.cancer.org/content/dam/cancer-org/research/cancer-facts-and-statistics/colorectal-cancer-facts-and-figures/colorectal-cancer-facts-and-figures-2017-2019.pdf

17. Moore S, Weiderpas E, Sampson J, Kitahara C, Keadle $\mathrm{S}$, Arem $\mathrm{H}$, et al. Association of Leisure-Time Physical Activity With Risk of 26 Types of Cancer in 1.44 Million Adults. JAMA Intern. Med. 2016; 176(6): 816-25. doi:10.1001/jamainternmed.2016.1548

18. Hormiga CM, León MH, Otero JA, Rodríguez LA. Factores de riesgo para enfermedades crónicas en Santander. Método STEP wise [internet].Bucaramanga (Colombia): Secretaría de Salud de Santander, Observatorio de Salud Pública de Santander; 2010 [citado marzo 2018]. Recuperado a partir de: https://www. who.int/ncds/surveillance/steps/2010 STEPS Survey_Colombia.pdf

19. Wolf A, Fontham E, Church T, Flowers C, Guerra C, LaMonte S, et al. Colorectal Cancer Screening for Average-Risk Adults: 2018 Guideline Update From the American Cancer Society. CA Cancer J Clin. 2018;00 (00) 1-32. doi: 10.3322/ caac. 21457.

20. Gil FL, Amaya MT, Riveros SV, Castaño R, Ibáñez $\mathrm{H}$, Huertas MM et al. Guía de práctica clínica para la tamización del cáncer colorrectal - 2015. Asociaciones Colombianas de Gastroenterología, Endoscopia digestiva, Coloproctología y Hepatología [internet]. 2018 [citado marzo 2018]. Recuperado a partir de: http://www.scielo.org.co/pdf/rcg/v30s1/v30s1a08. pdf 